ISSN electrónico: $1885-5210$

DOI: https://doi.org/10.14201/rmc202016e511520

\title{
5 PELÍCULAS PARA 5 PANDEMIAS
}

\section{Movies for 5 Pandemics}

María GARCÍA MORO; ; Matías ASTROZA RODRÍGUEZ ${ }^{\text {; }}$ Enrique GARCÍA MERINO;; Enrique GARCÍA SÁNCHEZ d,e,f; Jose Elías GARCÍA SÁNCHEZ ${ }^{\mathrm{d}, e, f}$

aUniversidad Nebrija. Madrid (España). ${ }^{b}$ Instituto Quipú. Madrid (España). 'Departamento de Sanidad, IES Martínez Uribarri, Salamanca (España). 'Departamento de Ciencias Biomédicas y del Diagnóstico. Facultad de Medicina. Universidad de Salamanca (España). eIUCE (Instituto Universitario de Ciencias de la Educación).

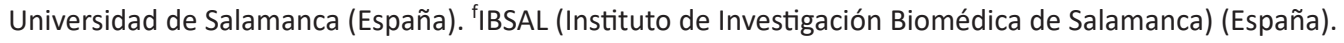

Correo electrónico: mgarciam@usal.es

Fecha de recepción: 25 de septiembre de 2020

Fecha de aceptación: 29 de septiembre de 2020

Fecha de publicación: 29 de enero de 2021

A lo largo de la historia diferentes pandemias han asolado a la humanidad. De todas ellas, salvo de la Covid-19, pues aún no ha habido tiempo de llevarla a la gran pantalla, ha habido numerosas representaciones en el cine comercial, algunas muy relevantes desde el punto de vista de la calidad cinematográfica y de la calidad científica con la que están documentados los guiones. Sería inalcanzable enumerar todas y cada una de ellas. Por tanto, en este artículo se plantea ofrecer a los lectores un ejemplo de cada una de estas pandemias (Peste, Viruela, Cólera, Gripe y VIH) que pueden ser útiles para la docencia y para la educación sanitaria de la población.
PESTE

Título original: La peste.

Otros títulos: The Plague.

País: Argentina, Francia, Gran Bretaña.

Año: 1992.

Director: Luis Puenzo.

Música: Vangelis.

Fotografía: Félix Monti.

Montaje: Juan Carlos Macías.

Guion: Luis Puenzo, basado en la novela homónima de Albert Camus.

Intérpretes: William Hurt, Sandrine Bonnaire, Jean-Marc Barr, Robert Duvall, Raúl Julia, Jorge Luz, Victoria Tennant, Atilio Veronelli, 
Francisco Cocuzza, Laura Palmucci, Norman Erlich, Marcos Woinsky, Duilio Marzio, Pancho Ibáñez, Horacio Fontova...

Color: color.

Duración: 142 minutos.

Género: drama.

Idioma original: inglés.

Sinopsis: "La peste muestra cómo se afecta la vida en una ciudad tras ser declarada una epidemia de peste. Pero va mucho más allá y refleja como el desastre y la desgracia pueden hacer aflorar los mejores sentimientos y actitudes de las personas para luchar y lograr sobreponerse ante lo que consideran injusto. El protagonista, el doctor Rieux, se queda en la ciudad porque marcharse sería desertar, siente la necesidad de combatir para acabar con el mal, que tiene la forma de la temible peste bubónica. Esta enfermedad da al traste con las vidas e ilusiones de cientos de inocentes. El miedo inunda las calles, y, además del doctor Rieux, hay otras personas dispuestas a ayudar y conseguir terminar con el horror. El esfuerzo común logra que la peste acabe desapareciendo ante el júbilo de todos. Pero queda planteada la duda sobre si no es posible que la amenaza siga vigente y algún día regrese (FILMAFFINITY)".

Productoras: Compagnie Française Cinématographique, The Pepper Prince Company Ltd., Oscar Kramer S. A., en asociación con Cinemania y con la participación de Canal+.

Disponibilidad: DVD.

Enlaces:

https://www.filmaffinity.com/es/film648316. html

https://www.imdb.com/title/tt0105127/ Trailer

Algunas películas en las que se aborda la peste de alguna forma (mención, presencia puntual o elemento argumental):

¿Por quién doblan las campanas? / For Whom the Bell Tolls (1943);; Ambiciosa / Forever Amber
(1947); Andrei Rublev (1966); Ave María (1999); Black Death (Garra negra) / Black Death (2010); Camino de la jungla / The Spiral Road (1962); Cinco tumbas para un medium / 5 tombe per un médium (1966); Colmillo blanco / White Fang (1991); Dager fra 100 ar (1970); De sade (1969); Descalzos por el parque / Barefoot in the Park (1967); Die pest in Florenz (1919); El álamo / The Alamo (1960); El aliento del diablo 1993; El año de la peste (1979); El caballero enmascarado / L'invincibile cavaliere mascherato (1963); El decameron / II Decamerone (1971); El despertar de Dracula / Dracula Rising (1993); El doctor Arrowsmith Arrowsmith (1931); El flautista / The Pied Piper (1972); El hijo de la evolución / Evolution's Child (1999); El hombre de la máscara de hierro / The Man in the Iron Mask (1997); El séptimo sello / Det Sjunde inseglet (1956); El talismán / King Richard and the Crusaders (1954); El último acto / All Is True (2018); El último cazador de brujas / The Last Witch Hunter (2015); El último valle / The Last Valley (1970); Extramuros (1985); Extraño suceso / So Long at the Fair (1950); Fausto / Faust (1926); Four frightened people (1934); Flukt: En los tiempos de la peste / Flukt (2012); Gladiator (2000); I love you (1918); Inquisición (1977); Join the marines (1937); Kafka, la verdad oculta / Kafka (1990); Kill the man (1999); Krabat y el molino del diablo / Krabat (2008) La armada Brancaleone / L'Armata Brancaleone (1965); La casa chica (1949); La cigarra no es un bicho (1964); La diligencia / Stagecoach (1939); La fuerza de un ser menor / Dominick and Eugene (1988); La isla de los muertos / Isle of the Dead (1945); La lista de Schindler / Schindler's List (1993); La mansión de Sangaree / Sangaree (1953); La máscara de la muerte roja / The Masque of the Red Death (1964); La mies es mucha (1949); La nueva cigarra (1976); La peste (1992); La plaga / Trollsyn (1994); La veneciana/ La venexiana (1985); Lanza rota / Broken Lance (1954); Las dos huérfanas / Orphans of the Storm (1921); Las locas, locas aventuras de Robin Hood / Robin Hood: Men In

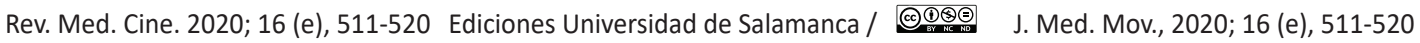
[512] 
Tights (1993); Lionheart (1987); Los caballeros de la mesa cuadrada y sus locos seguidores / Monty Python and the Holy Grail (1974); Los demonios / The Devils (1971); Los ritos satánicos de Drácula / The Satanic Rites of Dracula (1973); Los señores del acero / Flesh and Blood (1985); Los últimos de Filipinas (1945); Mar de luna (1994); Más fuerte que su destino / Dangerous Beauty (1998); Masque of the red death (1989); Masque of the red death (1990); Midnight warning (1932); Mixed blood (1916); Monsieur Vincent (1947); Navigator: una odisea en el tiempo / The Navigator: A Mediaeval Odyssey (1988); Nazarin (1958); Niezwykla podróz baltazara kobera (1988); No escape (1934); Nosferatu el vampiro / Die Zwoelfte Stunde; Nosferatu, eine Symphonie des Grauens (1922); Nosferatu, el príncipe de las tinieblas / Nosferatu a Venezia (1988); Nosferatu, vampiro de noche / Nosferatu: Phantom der Nacht; Nosferatu: The Vampire (1978); Nostradamus (1994); Out of the darkness (1985); Pánico en las calles / Panic in the Streets (1950); Peste negra / Black Death (1992); Piratas / Pirates (1986); Plaga final / Pars vite et reviens tard (2007) Project X (1968); Restauración / Restoration (1995); Ridicule. Nadie está a salvo Ridicule (1996); Shakespeare enamorado / Shakespeare in Love (1998); Singoalla (1949); Sombras blancas / White Shadows in the South Seas (1928); Sonhos tropicais (2001); Suspect (1960); TaiPan (1986); The Golem (2018); The lost people (1949); The plague dogs (1982); Todo lo que siempre quiso saber sobre el sexo pero jamás se atrevió a preguntar / Everything You Always Wanted to Know About Sex But Were Afraid to Ask (1972); Troya / Troy (2004); Vinieron las Iluvias The Rains Came (1939); Yo, la peor de todas (1990).
Acción: Orán (Sur de Sudamérica). Última década del siglo XX.



VIRUELA

Título: La puta del rey.

Título original: La putain du roi.

Otros títulos: The King's Whore (Estados Unidos), La puttana del re (Italia), La prostituta del rey (Argentina), A Amante do Rei (Brasil).

País: Francia, Austria, Italia.

Año: 1990.

Director: Alex Corti.

Música: Gabriel Yared

Fotografía: Gernot Roll.

Montaje: Bryan Oates, Joële Van Effenterre. 
Guion: Daniel Vigne, Frederic Rápale, Axel Corti (basado en la novela homónima de Jacques Tournier).

Intérpretes: Valeria Golino, Timothy Dalton, Robin Renucci, Margarte Tyzack, Stephane Freiss, Eleanor David, Paul Crauchet, Amy Werba, Franco Valobra, Anna Bonaiuto, Feodor Chaliapin Jr., Lea Padovani, Caterina Vertova, Federico Pacifici,...

Color: color.

Duración: 93 minutos.

Género: drama histórico.

Idioma original: inglés, francés.

Sinopsis: "Finales del siglo XVII. El rey Vittorio Amadeo se encapricha apasionadamente de la esposa de su chambelán, la duquesa de Luynes. Ella, que ama a su marido, le rechaza, pero el esposo se la ofrece a su señor. La unión de los dos se ve forzada por el odio y el deseo de herirse mutuamente. Las cosas van a cambiar, pero ya es demasiado tarde (FILMAFFINITY)".

Productoras: AFC, FR3 Films Productions, Soficas Sofinergie, Images Investissements, Umbrella Films,...

Premios: Nominada a la Palma de Oro a la mejor película en el Festival de Cannes (1990).

Disponibilidad: DVD.

Enlaces:

https://www.filmaffinity.com/es/film152617. html

https://www.imdb.com/title/tt0100440 Tráiler en versión original

Algunas películas en las que se aborda la viruela de alguna forma (mención, presencia puntual o elemento argumental):

22 ángeles (2016); 80000 suspects (1963); A matter of who (1961); Adventure island (1947); Amenaza biológica / Doomsday Man (2000); Arizona stage coach (1942); Ave Maria (1999); Battles of chief Pontiac (1952); Battling marshal (1950); Blood arrow (1958); Camino de la jungla / The Spiral Road (1962); Camino de Oregon / The Way West (1967); Cambio de reinas/ L'echange de princesses (2017); Charlie chan's greatest case (1933); Cuando hierve la sangre / Never So Few (1959); Cyclone fury (1951); Danger within (1918); Doctor Akagi / Kanzo sensei (1998); Down to earth (1917); Ebb tide (1937); El pacto de los lobos / Le pacte des loups (2001); El padrino / The Godfather (1972); El político / All the King's Men (1949); El puente de Casandra / The Cassandra Crossing (1976); El puente de San Luis Rey / The Bridge of San Luis Rey (2004); El viajero solitario / One man's journey (1933); Frankenstein de Mary Shelley / Mary Shelley's Frankenstein (1994); Fish hawk (1979); Halcón de invierno / Winterhawk (1975); Hearts of oak (1914); Historia de filadelfia / The Philadelphia Story (1940); It's a gift (1934); Kali yug (1963); La gran ilusión / La Grande illusion (1937); La saga de Gösta Berling / Gösta Berlings saga (1924); Las amistades peligrosas / Dangerous Liaisons (1988); Las montañas de la luna / Mountains of the Moon (1990); Le jour avant le lendemain (2008); Los dos sargentos / I due sergenti (1936); Los generales de la arena / The Sandpit Generals (1971); Los tres mosqueteros (los diamantes de la reina) / The Three Musketeers (1973); Los últimos días del edén / Medicine Man (1992); Manto negro / Black Robe (1991); María Antonieta / Marie Antoinette (2006); Moll flanders, el coraje de una mujer / Moll flanders (1996); My Antonia (1995); Misión Imposible: Fallout / Mission: Impossible - Fallout (2018); My dear miss aldrich (1937); Noche de angustia / Vigil in the Night (1940); Pasteur (1935); Quarantined rivals (1927); Retaguardia / The Command (1954); Señor Skeffington / Mr. Skeffington (1944); Seven days (1925); Sin control / Derailed (2002); Sin perdón / Unforgiven (1992); Smallpox 2002: Silent Weapon (2002); Sonhos 
tropicais (2001); Stagecoach to dancers' rock (1962); Tales from the gimli hospital (1988); The devil's apple tree (1929); The good for nothing (1914); The killer that stalked New York (1950); The man from Dakota (1940); The woman in politics (1916); Tragedia y triunfo de Verdi / Giuseppe Verdi (1950); Trails of the golden west (1931); Un asunto real / En kongelig affære (2012); Valmont (1989); Vámonos con Pancho Villa (1936); Variola vera (1981); Young Catherine (1991); Your best friend (1922); Zaraza (1972).

Acción: Piamonte (Italia). Finales del siglo XVII.

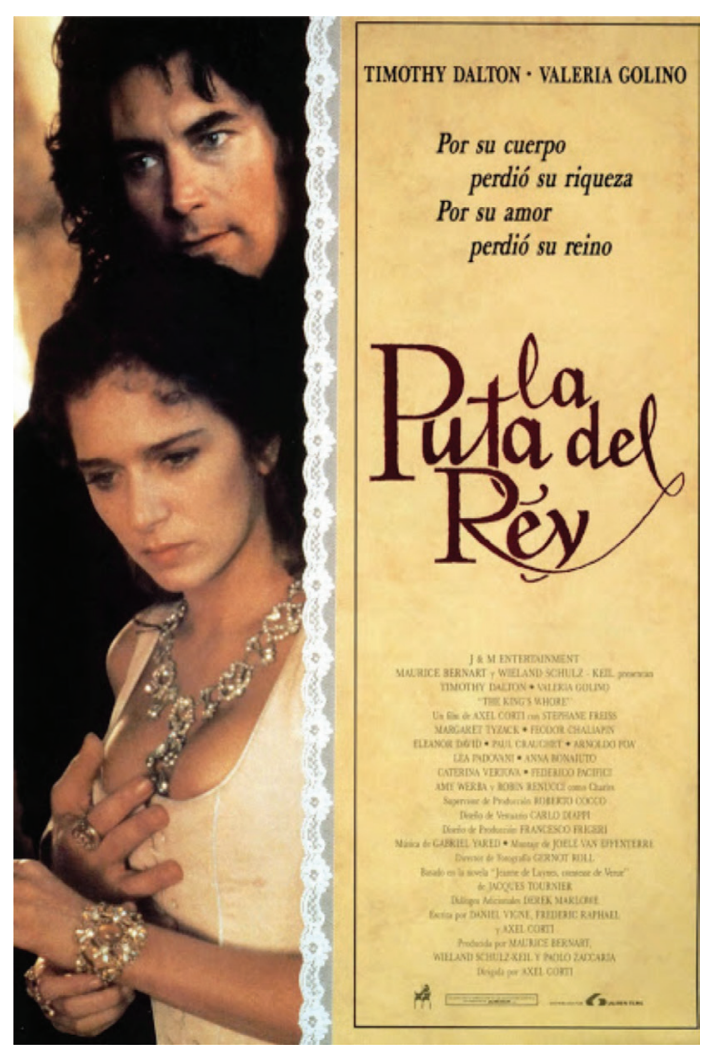

Cartel español.

\section{CóleRA}

Título: El velo pintado.

Título original: The painted veil.

Otros títulos: Al otro lado del mundo (Argentina).

País: China, USA, Canadá.

Año: 2006.

Director: John Curran.

Música: Alexandre Desplat.

Fotografía: Stuart Dryburgh.

Montaje: Alexandre de Franceschi.

Guion: Ron Nyswaner badao en la novela homónima de W. Somerset Maugham.

Intérpretes: Naomi Watts, Edward Norton, Liev Schreiber, Catherine An, Bin Li, Bin Wu, Alan David, Marie-Laure Descoreaux, Sally Hawkins, Juliet Howland, Toby Jones, Lorraine Laurence, Johnny Lee, Li Feng, Gesang Meiduo, Ian Renwick, Diana Rigg, Cheng Shihan, Maggie Steed, Lucy Voller, Anthony Wong Chau-Sang...

Color: color.

Duración: 125 minutos.

Género: drama, romance.

Idioma original: inglés.

Sinopsis: "Años veinte. Kitty (Naomi Watts) es una joven inglesa de la alta sociedad que, para escapar de su ambiente familiar, se casa con Walter (Edward Norton), un prometedor médico. El matrimonio se traslada a Shangai, donde Kitty tiene una aventura con un norteamericano (Liev Schreiber). Cuando Walter descubre la infidelidad de su esposa, decide trasladarse con ella a una de las regiones más remotas de China, para intentar luchar contra una mortal epidemia de cólera (Filmaffinity)".

Productoras: WIP, Stratus Film Co., Bob Yari Productions, Class 5 Films, The Colleton Company, Dragon Studios, Emotion Pictures, The Mark Gordon Company, Warner China Film HG Corporation.

Premios: Globo de Oro a la Mejor Banda Sonora en 2007.

Disponiblidad: DVD y Blu-ray. 


\section{Enlaces:}

http://www.filmaffinity.com/es/film406338. html

http://www.imdb.com/title/tt0446755 Trailer

Algunas películas en las que se aborda el cólera de alguna forma (mención, presencia puntual o elemento argumental):

Al Youm El Sades (1986); Ana y el rey / Anna and the King (1999); Baby, el secreto de una leyenda perdida / Baby... Secret of the Lost Legend (1985); Bandera amarilla / Vessel of Wrath (1938); Camino de la jungla / The Spiral Road (1962); Caravana de mujeres / Westward the Women (1951); Carry on up the khyber (1968); Contagio / Contagious (1998); Contaminación / No Blade of Grass (1970); Doc's kingdom (1987); Doctor (1940); Doctor akagi / kanzo sensei (1998); Egypt by three (1953); El amor en los tiempos del cólera / Love in the Time of Cholera (2007); El bárbaro y la geisha / The Barbarian and the Geisha (1958); El husar en el tejado / Le hussard sur le toit (1995); El jardín secreto (1949) / The Secret Garden (1949) (1949); El jardín secreto (1987) / The Secret Garden (1987) (1987); El séptimo pecado / The Seventh Sin (1957); El vagabundo de las islas / The Beachcomber (1954); El velo pintado / The Painted Veil (1934); Frankenstein de Mary Shelley / Mary Shelley's Frankenstein (1994); House on the hill (1970); Inside straight (1951); La fuerza de un ángel / Entertaining Angels: The Dorothy Day Story (1996); La hermana blanca / The White Sister (1933); La institutriz / The Governess (1998); La jungla en armas / The Real Glory (1939); La novicia / Storia di una Capinera (1994); La reina de Nueva York / Nothing Sacred (1937); La senda de los elefantes / Elephant Walk (1954); La última carga / The Charge of the Light Brigade (1968); Lejos de Liverpool / The Leaving of Liverpool (1992); Lluvia / Rain (1932); Los desesperados (1969); Luz a oriente / Oil for the Lamps of China (1935); Mens pesten raser (1913);
Muerte en Venecia / Morte a Venezia (1971); Pacific liner (1938); Pánico en las calles / Panic in the Streets (1950); Pasaje a la India / A Passage to India (1984); Por quién doblan las campanas / For Whom the Bell Tolls (1943); Regreso al lago azul / Return to the Blue Lagoon (1991); Salto a la gloria (1959); Siete mujeres / Seven Women (1966); Song of my heart (1948); Stronger than death (1920); The beggar of cawnpole (1916); The blonde saint (1926); The isle of life (1916); The lady with a lamp (1951); The light ahead (1939); The little shepherd of kingdom come (1961); The safety curtain (1918); The secret garden (1919); The secret garden (1975); The silver darlings (1947); The white angel (1936); Un aventurero audaz / Bulldog Drummond Strikes Back (1934); Unfaithful wife (1915); Wilson's reward (1980); Without benefit of clergy (1921); Youthful cheaters (1923).

Acción: China. Años 20 del siglo XX.

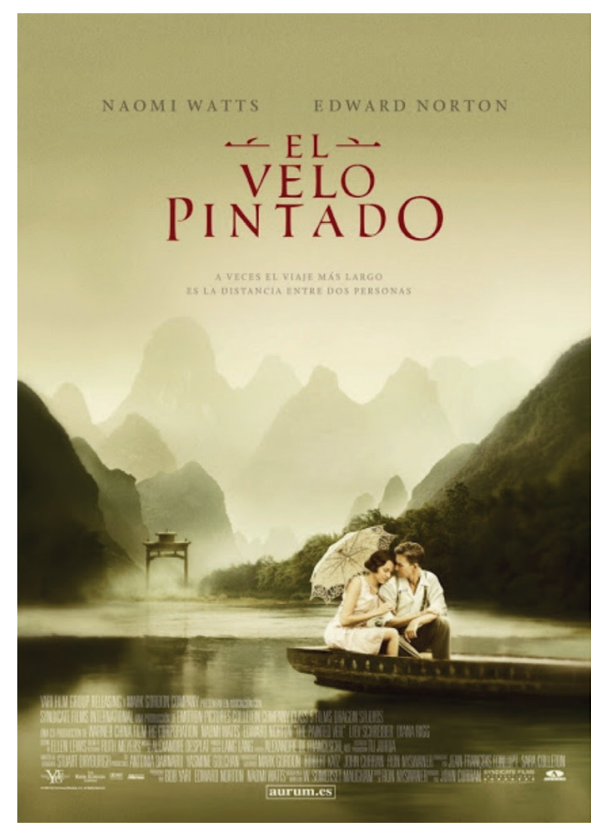

Cartel español. 


\section{GRIPE}

Título original: Spanish Flu The Forgotten Fallen. (Película realizada para la TV).

Otros títulos: Spanyolnátha: Az elfeledett esetek (Hungría), Spanska sjukan (Suecia).

País: Reino Unido.

Año: 2009.

Director: Justin Hardy.

Fotografía: Douglas Hartington.

Montaje: Alastair Reid.

Guion: Peter Guinness, Peter Harness.

Intérpretes: Bill Paterson, Mark Gatiss, Charlotte Riley, Kate Ambler, John Branwell, Raffey Cassidy, Kenneth Cranham, John Draycott, Gerard Fletcher, Paul Freeman, Olwen May, Jack McMullen, Ian Mercer, Martin Oldfield, Sarah Parks, Will Tacey.

Color: color y blanco y negro.

Duración: 60 minutos.

Género: drama, biográfico, sanitario.

Idioma original: inglés.

Sinopsis: la acción comienza en el mes de noviembre de 1918 y se desarrolla durante los primeros meses después Primera Guerra Mundial. Se centra en la actuación del Dr James Niven en la segunda ola de la gripe española que azotó Manchester.

Productora: Hardy Pictures, Hardy and Sons. Disponibilidad: DVD.

Enlaces:

https://www.imdb.com/title/tt1473802 Tráiler en versión original

Algunas películas en las que se aborda la gripe de alguna forma (mención, presencia puntual o elemento argumental):

1918 (1985); 2001 una odisea en el espacio /2001: A Space Odyssey (1968); Aeropuerto 78 / SST: Death Flight (1977); Amenaza biológica / Doomsday Man (2000); Amigo/amado (1998); Canción de invierno / Sarah, Plain and
Tall: Winter's End (1999); Carry on constable (1960); Confesiones verdaderas / True Confessions (1981); Contagio mortal / Le neuvième jour (1995); Crimen misterioso / The Darklings (1999); Descalzos por el parque / Barefoot in the Park (1967); Despertares / Awakenings (1990); Doctor Akagi / Kanzo sensei (1998); EI fantasma de High River Sightings: Heartland Ghost (2002); El jardín de la alegría / Saving Grace (2000); El juez / L'hermine (2015); El país del agua / Waterland (1992); El reloj de pandora / Pandora's Clock (1996); El virus perdido / Runaway Virus (2000); En la línea de fuego / In the Line of Fire (1993); Exterminio (virus) / Fukkatsu no hi (1980); Gas attack (2001); Gélidas escenas invernales / Head Over Heels (1979); Goodbye miss 4th of july (1988); Gripe Aviar: Virus mortal / Virus au paradis (2003); Jugando en los campos del señor / At Play in the Fields of the Lord (1991); La batalla de Midway / Midway (1976); La canción de Bernadette / The Song of Bernadette (1943); La colina de la felicidad I'd Climb the Highest Mountain (1951); La fuerza de un ángel / Entertaining Angels: The Dorothy Day Story (1996); La naranja mecánica / A Clockwork Orange (1971); Los últimos días del edén / Medicine Man (1992); Misión imposible 2 / Mission: Impossible-2 (2000); Pelham 1, 2, 3 The Taking of Pelham One Two Three (1974); Pepe (1960); Perdidos en el espacio / Lost in Space (1998); Qué bello es vivir It's a Wonderful Life (1946); Rutas infernales / Three faces west (1940); South of heaven, west of hell (2000); Testamento de juventud/ Testament of Youth (2014); The man in the attic (1995); The reverse be my lot (1937); Tres deseos / Three Wishes (1995); Tundra (1936); Virus / Gamgi (2013); Virus mortal / Fatal Contact: Bird Flu in America (2006).

Acción: Manchester 1918. 


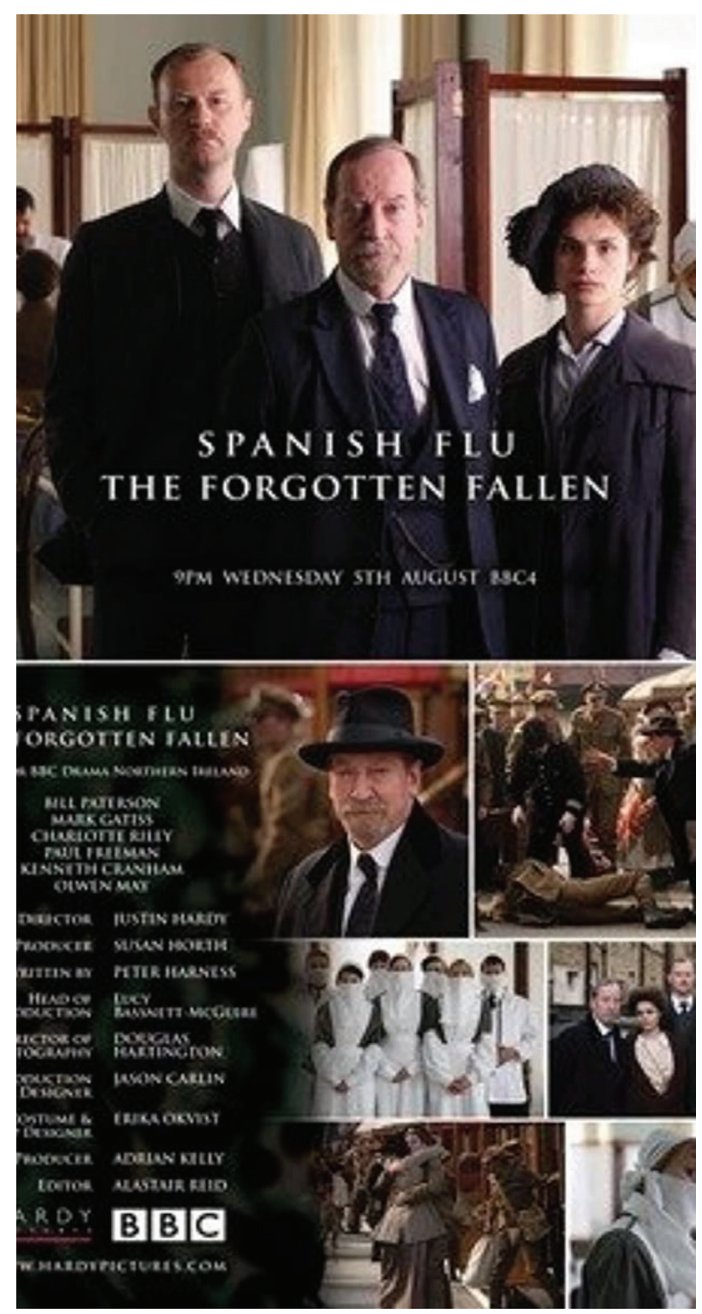

Cartel inglés.

VIH / SIDA

Título: En el filo de la duda (España) / Y la banda siguió tocando (Latinoamérica).

Título original: And the band played on (película realizada para TV).

País: Estados Unidos.

Año: 1993.

Director: Roger Spottiswoode.

Música: Carter Burwell.
Fotografía: Paul Elliott.

Montaje: Lois Freeman-Fox.

Guion: Arnold Schulman (Basado en la novela de Randy Shilts).

Intérpretes: Matthew Modine, Alan Alda, Richard Gere, Phil Collins, Patrick Bauchau, David Dukes, Anjelica Huston, Steve Martin, Swoosie Kurtz, Richard Jenkins, Glenne Headly, Ian McKellen, Lily Tomlin, Nathalie Baye, Charles Martin Smith, ...

Color: color.

Duración: 140 minutos.

Género: drama, enfermedad, sida, telefilm.

Idioma original: inglés.

Sinopsis: "Minucioso relato de las reacciones sociales ante el descubrimiento del SIDA y el tenso trabajo de los profesionales y médicos que lo investigan (Filmaffinity)".

Productoras: HBO; Odissey Entertainment; SpeIling Entertainment.

Premios: 14 nominaciones y 3 premios Emmy (1994) incluyendo mejor telefilm y casting. Globos de oro 1993: nominada Mejor miniserie o película para TV y actor principal.

Disponibilidad: DVD.

\section{Enlaces:}

h t t p s:// w w w. im d b.com/title / tt0106273/?ref_=fn_al_tt_1

https://www.filmaffinity.com/es/film201278. html

\section{Trailer en inglés}

Algunas películas en las que se aborda el VIH/SIDA de alguna forma (mención, presencia puntual o elemento argumental):

120 pulsaciones por minuto / 120 battements par minute (2017); 3 agujas / 3 Needles (2005); A causa secreta (1994); A death in the family (1986); A la belle etoile (1993); A mort la mort! (1999); A return to salem's lot (1987); Afrique, mon afrique (1995); Aids- die schleichende gefahr (1985); Al caer la noche / In the Gloaming (1997); Algo casi perfecto / The 
Next Best Thing (2000); Algún lugar / A Place for Annie (1994); Alive \& kicking (1996); American adobo (2001); American riscio (1990); Amor fatal / Fatal love (1990); Amor que mata (1992); Amores (1998); An early frost (1985); Andre's mother (1990); Antes que anochezca / Before Night Falls (2000); Around the fire (1999); As is (1986); At risk (1994); Ausgerechnet zoé (1994); Azúcar amarga (1996); Belleza robada (1995); Bienvenido-welcome (1994); Bocados de realidad / Reality Bites (1994); Boys in love (1996); Buddies (1985); Bullet on a wire (1998); Camina hacia la luz / Go toward the light (1989); Carandiru (2003); Chocolate babies (1996); Ciudadano Cohn / Citizen Cohn (1992); Clockers: camellos / Clockers (1995); Compañeros inseparables / Longtime Companion (1990); Con mis ojos cerrados / Close My Eyes (1991); Contratiempos / Grief (1993); Crocodile tears (1998); Cuernos de espum / Shampoo horns (1996); Dallas Buyers Club (2013); Dejando metrópolis / Leaving Metropolis (2002); Del otro lado (1999); Después de una noche / One night stand (1997); Drôle de Felix (2000); Dulce Jane / Sweet Jane (1998); Eine mutter kämpft um ihren sohn (1994); En una noche de luna / Clair; In una notte di chiaro di luna (1989); Energía descarriada / Nervous Energy (1995); Facteur VIII (1994); Fiesta de despedida/It's my party (1996); Fotos del alma (1995); Gefahr für die liebe-aids (1985); Gia (1997); Giorni (2001); Hessed mufla (1992); J'ai horreur de l'amour (1997); Jeanne y el chico formidable / Jeanne et le garçon formidable (1998); Kosumosu (1997); L'homme que j'aime (1997); La banda del listón rojo / Red Ribbon Blues (1995); La cara del crimen/ The Killing Machine (1994); La historia de Ryan White / The Ryan White Story (1989); La niña de tus sueños (1995); La súplica de una madre / A Mother's Prayer (1995); La velocidad de la vida / The Velocity of Gary (1998); La vida alegre (1987); Las horas / The Hours (2002); Las noches salvajes / Les Nuits fauves (1992);
Lazos (1993); Lazos de sangre / My Brother's Keeper (1995); Le traite du hasard (1998); Les passangers (1998); Lisa (1996); Los amigos de Peter / Peter's Friends (1992); Love, valour and compassion (1996); Men in love (1989); Mentira /Mensonge (1993); Merci la vie (1991); Mes 17 ans (1996); Mi propia lucha / My Own Country (1998); Mute love (1999); No blame (1988); No olvides que vas a morir / $N$ ' oublie pas que tu vas mourir (1995); Nungameinun boinun saesang (1995); One week (2000); Paradise framed (1995); Pase lo que pase / Roommates (1994); Pequeñas víctimas / The Littlest Victims (1989); Philadelphia (1993); Pora na czarownice (1993); Punto ciego /Slepa Pega (2002); Que nada nos separe / The Cure (1995); Que nadie duerma / No One Sleeps (2000); Rent: Filmed Live on

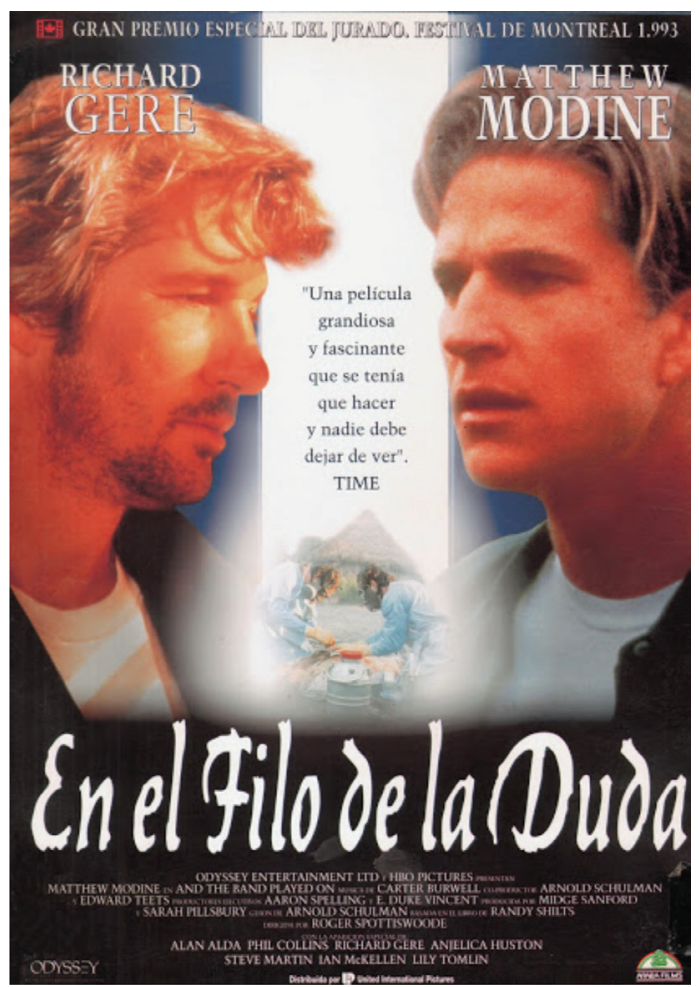

Cartel español. 


\section{PELÍCULAS PARA 5 PANDEMIAS}

MARÍA GARCÍA MORO; MATÍAS ASTROZA RODRÍGUEZ; ENRIQUE GARCÍA MERINO; ENRIQUE GARCÍA SÁNCHEZ; JOSE ELIIAS GARCÍA SÁNCHEZ

Broadway (2008); Rompiendo la superficie / Breaking the Surface: The Greg Louganis Story (1996); Rosa roth-lügen (1995); Sabine (1993); Saigon baby (1995); Siempre juntos (Todos contra Leo) / Tout contre Léo (Close to Leo) (2002); Sólo con tu pareja (1991); Soy positive / Sono Positivo (1999); Sweet as you are (1987); Te estoy perdiendo /I' m Losing You (1998); Test (2013); The event (2003); The last supper (2004); The love session(1995); The night larry kramer kissed me (2000); The perfect son(2000); The unknown cyclist (1997); The weekend (1999); Tiempo de esperanza / And Then There Was One(1994); Touch me (1997); Trainspotting (1996); Últimos días en La Habana (2016); Un año sin amor (2005); Un virus no conoce moral / Ein Virus kennt keine Moral (1985); Via appia (1990); Xian fa zhi ren (1990); Yesterday (2004); Zero patience (1994); Zinda gi zindabad (1998). Acción: Estados Unidos. Años 80 del siglo XX. 